\title{
Refractive index measurement of the mouse crystalline lens using optical coherence tomography
}

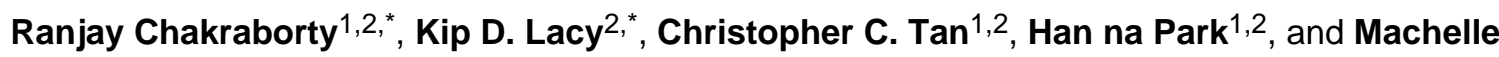 \\ T. Pardue ${ }^{1,2}$ \\ ${ }^{1}$ Department of Ophthalmology, Emory University School of Medicine, 1365B Clifton Rd NE \\ Atlanta, GA 30322 \\ ${ }^{2}$ Center of Excellence in Visual and Neurocognitive Rehabilitation, Atlanta VA Medical Center, \\ 1670 Clairmont Road, Decatur, GA 30033
}

\section{Abstract}

In recent years, there has been a growing interest for using mouse models in refractive development and myopia research. The crystalline lens is a critical optical component of the mouse eye that occupies greater than 50\% of the ocular space, and significant increases in thickness with age. However, changes in refractive index of the mouse crystalline lens are less known. In this study, we examined the changes in thickness and refractive index of the mouse crystalline lens for two different strains, wild-type (WT) and a nyx mutant (nob) over the course of normal visual development or after form deprivation. Refractive index and lens thickness measurements were made on ex vivo lens using spectral domain optical coherence tomography (SD-OCT). Comparison of refractive index measurements on 5 standard ball lenses using the SDOCT and their known refractive indices (manufacturer provided) indicated good precision (intraclass correlation coefficient, 0.998 and Bland-Altman coefficient of repeatability, 0.116) of the SD-OCT to calculate mouse lens refractive index ex vivo. During normal visual development, lens thickness increased significantly with age for three different cohorts of mice, aged 4 (average thickness from both eyes; WT: $1.78 \pm 0.03$, nob: $1.79 \pm 0.08 \mathrm{~mm}$ ), 10 (WT: $2.02 \pm 0.05$, nob: 2.01 $\pm 0.04 \mathrm{~mm}$ ) and 16 weeks (WT: $2.12 \pm 0.06, n o b: 2.09 \pm 0.06 \mathrm{~mm}, \mathrm{p}<0.001$ ). Lens thickness was not significantly different between the two strains at any age $(\mathrm{p}=0.557)$. For mice with normal vision, refractive index for isolated crystalline lenses in nob mice was significantly greater than WT mice (mean for all ages; WT: $1.42 \pm 0.01$, nob: $1.44 \pm 0.001$, p<0.001). After 4 weeks of form deprivation to the right eye using a skull-mounted goggling apparatus, a thinning of the crystalline lens was observed in both right and left eyes of goggled animals compared to their naïve controls (average from both the right and the left eye) for both strains $(\mathrm{p}=0.052)$. In form deprived mice, lens refractive index was significantly different between the goggled animals and non-goggled naïve controls in nob mice, but not in WT mice ( $\mathrm{p}=0.009)$. Both eyes of goggled nob mice had

Corresponding author: Machelle T. Pardue, PhD, Department of Ophthalmology, Emory University School of Medicine, 1365B Clifton Rd NE Atlanta, GA 30322, mpardue@emory.edu, Phone:1-404-321-6111X17342.

These authors had equal contributions to this work

Publisher's Disclaimer: This is a PDF file of an unedited manuscript that has been accepted for publication. As a service to our customers we are providing this early version of the manuscript. The manuscript will undergo copyediting, typesetting, and review of the resulting proof before it is published in its final citable form. Please note that during the production process errors may be discovered which could affect the content, and all legal disclaimers that apply to the journal pertain. 
significantly greater lens refractive index (goggled, $1.49 \pm 0.01$; opposite, $1.47 \pm 0.03$ ) compared to their naïve controls $(1.45 \pm 0.02, \mathrm{p}<0.05)$. The results presented here suggest that there are genetic differences in the crystalline lens refractive index of the mouse eye, and that the lens refractive index in mice significantly increase with form deprivation. Research applications requiring precise optical measurements of the mouse eye should take these lens refractive indices into account when interpreting SD-OCT data.

\section{Keywords}

refractive index; geometrical thickness; crystalline lens; optical coherence tomography; mouse; myopia

\section{Introduction}

During normal refractive development, the axial length of the eye and the refractive power of the optical components (primarily the cornea and the crystalline lens) precisely match to produce the ideal refractive state of emmetropia (Wallman and Winawer, 2004). In emmetropia, the focal plane is located at the photoreceptor inner segments (Crewther, 2000). Although axial length has been largely identified as the primary biometric determinant of refractive growth (Gordon and Donzis, 1985, Mutti et al., 2005), the crystalline lens also plays an important role in emmetropization of human eyes (Mutti et al., 1998). Whilst the crystalline lens in human eyes undergoes a concurrent thinning and flattening with age (Mutti et al., 1998; Mutti et al., 2005; Zadnik et al., 1995), different mammals show both increase (tree shrew, Norton and McBrien, 1992; guinea pigs, Zhou et al., 2006; mouse, Zhou et al., 2008) and decrease (marmoset, Troilo and Judge, 1993) in lens thickness during normal eye development. Previous cross-sectional studies have reported changes in the equivalent refractive index of the crystalline lens with age in children (Garner et al., 1994; Mutti et al., 1991; Mutti et al., 1998); however, results between studies have been variable. There have been only limited investigations into developmental changes in lens refractive index of mammalian eyes.

Experiments on various animal models including chickens (Schaeffel et al., 1988; Wallman and Adams, 1987; Wallman et al., 1995), primates (Hung et al., 1995; Smith III and Hung, 2000; Troilo and Judge, 1993), fish (Shen et al., 2005), and mammals (Edwards, 1996) have been influential in understanding the potential mechanisms of refractive development of the eye (Siegwart and Norton, 2011; Wallman and Winawer, 2004; Wildsoet, 1997). In recent years, there has been a growing interest in using mouse models in refractive development and myopia research. In addition to a closer resemblance of their retinal structure to humans, the mouse model specifically offers an advantage of manipulating both genes and environment in the same animal using various knockout models (Pardue et al., 2013; Schaeffel 2008).

The crystalline lens is the most important optical component in mice, and occupies a large proportion (about 50-60\%) of the mouse eye (Pardue et al., 2013). Previous studies have reported a linear increase in lens thickness of C57BL/6 mice with age (Chou et al., 2011; Puk et al., 2006; Tkatchenko et al., 2010a; Schmucker and Schaeffel, 2004a; Zhou et al., 
2008), which is significantly correlated with the refractive development of the eye (Tkatchenko et al., 2010a; Zhou et al., 2008). Ocular dimensions of the mouse eye have been most successfully measured with either low or partial coherence interferometry (PCI, Park et al., 2012; Schmucker and Schaeffel, 2004a), or most recently with the optical coherence tomography (OCT, Jiang et al., 2012; Park et al., 2012; Wang et al., 2011; Zhou et al., 2008). Since measurement of optical parameters with optical interferometry techniques (such as PCI or OCT) require the calculation of geometric path length from optical path length using refractive indices of the eye, inaccuracies in the refractive index measurements of each ocular component, especially the crystalline lens, could significantly affect the geometric measurements from these techniques in rodent eyes. Remtulla and Hallett (1985) found the equivalent lens refractive index for an adult C57BL/6 mouse to be 1.659 using paraxial ray tracing from frozen sections (measured using Abbe refractometer and interference microscopy) for a wavelength of $655 \mathrm{~nm}$. In this study, sectioning artifacts or insufficient resolution from frozen sections may have influenced the values of measured radii of curvature. In a later study, using paraxial ray tracing for the same wavelength, Schmucker and Schaeffel (2004a) calculated the lens refractive index in the mouse eye to increase from 1.568 to 1.605 from age 22 to 100 days. Given the uncertainty in the reported values of lens refractive index by these studies; non-destructive and direct methods of measurement may provide a better model of refractive index in the mouse eye.

In this study, we calculated the refractive index of the isolated mouse crystalline lens using an indirect, spectral domain OCT (SD-OCT) imaging technique, as described by Uhlhorn et al (2008). Since small changes in lens refractive index with nuclear cataracts are known to produce corresponding significant myopic shifts in human eyes (Brown and Hill, 1987; Kuroda et al., 2002; Pesudovs and Elliott, 2003), differences in refractive index of the crystalline lens between strains or in response to visual deprivation could influence refractive error. Here, we studied mouse crystalline lens refractive index profiles to determine whether changes occur 1) across age, 2) due to gene mutations that increase myopia susceptibility, and 3) with form deprivation myopia.

\section{Material and methods}

\subsection{Animals and housing}

All mice used in this study were obtained from an in-house breeding colony located at the Atlanta Veterans Affairs Medical Center (VAMC). Male and female wild-type (WT) C57BL/6J mice (Jackson Laboratory, Bar Harbor, ME) and nob mice on a C57BL/6J background were housed in standard mouse cages with chow and water accessible ad libitum under a 12:12 hour light/dark cycle. Nob mice (Pardue et al., 1998) have a null mutation in $N y x$ (Gregg et al., 2003), which blocks visual transmission in the retinal ON pathway (Gregg et al., 2003; Pardue et al., 1998). Nob mice are highly susceptible to formdeprivation myopia compared to their wild-type counterparts (Pardue et al., 2008).

Changes in lens thickness and refractive index were measured for both mouse strains using SD-OCT at 4, 10, and 16 weeks during normal refractive development and after 4 weeks of form-deprivation using diffuser goggles (see below). All experiments were approved by the 
Institutional Animal Care and Use Committee at Atlanta VAMC and adhered to the ARVO statement for the Use of Animals in Ophthalmic and Vision Research.

\subsection{SD-OCT system}

We used a $1310 \mathrm{~nm}$ SD-OCT (Bioptigen Inc, Durham, NC, USA) to measure in vivo ocular dimensions and ex vivo crystalline lens thickness in mice. The SD-OCT contains a stationary reference mirror and a spectrometer that measures the light reflected from ocular structures as a function of the wavelength. The spectral trace then undergoes a Fourier transformation into the time domain in order to produce the depth information of ocular structures (Schmitt, 1999). This SD-OCT has been shown to provide a reliable measurement of optical components in the mouse eye (Park et al., 2012), with an axial resolution of $8 \mu \mathrm{m}$, and a standard deviation of $10 \mu \mathrm{m}$ on repeated measurements. The instrument has an imaging window of $4 \mathrm{~mm}$, and uses a "mirror artifact" to capture both the front and back of the eye superimposed on each other in a single frame (Park et al., 2012). The in vivo OCT measurements were performed under anesthesia [intraperitoneal injections of ketamine $(80 \mathrm{mg} / \mathrm{kg}) / x y l a z i n e(16 \mathrm{mg} / \mathrm{kg})]$, and 1 radial scan and 4 linear scans (each scan containing an average of 10 images) were acquired for each eye, as described in Park et al., 2012.

\subsection{Crystalline lens experiment and refractive index measurement}

After the in vivo images were acquired, mice were euthanized by cervical dislocation. Lenses were removed from the enucleated eyes of mice after death. Eyes were dissected while immersed in Dulbecco's Modified Eagle Medium (DMEM, Invitrogen, Carlsbad, CA, USA) solution to preserve ocular hydration (Augusteyn et al., 2006). Crystalline lenses were extracted by removing the sclera, and then gently removing the lens with a small portion of ciliary body remaining as a reference point when positioning the lens for the OCT imaging.

For lens refractive index measurements in the mouse eye, a lens measurement chamber was recreated (Figure 1a) based on Uhlhorn's model (2008) for isolated human crystalline lenses. The isolated mouse lens was placed inside a plastic petri dish $(35 \mathrm{~mm}$ height and 40 mm diameter) filled with DMEM solution. The inner surface of the petri dish was coated with a thin anti-reflection film to minimize unwanted reflections during imaging. The lens was placed on a rubber O-ring (1.42 $\mathrm{mm}$ internal diameter) at the center of the petri dish, such that the equator of the lens rested along the circumference of the O-ring. The effect of meniscus variability on imaging was avoided by imaging the lens at the center of the petri dish, as well as standardizing the amount of DMEM solution in the petri dish during each scan.

The OCT laser was vertically mounted over the lens measurement chamber to image the crystalline lenses along the optical axis. The lens axis was aligned normal to the OCT laser, producing a specular reflection from the anterior surface of the lens. Pilot studies conducted prior to the experiment suggested the refractive index to be equal, irrespective of whether the anterior or the posterior surface of the spherical crystalline lens (Rafferty and Goossens, $1978)$ is imaged. Five rectangular scans $(4 \mathrm{~mm} \times 4 \mathrm{~mm})$ were obtained along the optical axis for each lens. To avoid degradation of the lens over time, all lenses were imaged within $10-$ 
12 minutes of sacrificing the animal. Only clear isolated lenses with no apparent opacities in the OCT were used for calculating refractive index in this study.

In the lens measurement chamber, the laser beam from the OCT is differentially refracted by the medium in the chamber (DMEM) and the lens. If the optical lens thickness $(\tau)$ and optical displacement (or window distortion, $\delta$ ) of the light source when travelling through the isolated lens with reference to the chamber medium is known (Figure 1b), geometrical thickness $(t)$ of the lens can be calculated from the formula given by Uhlhorn et al (2008).

$$
t=\left(1 / n_{\mathrm{o}}\right)(\tau-\delta)
$$

In the above equation, the optical lens thickness $(\tau)$ along the lens axis was calculated from the OCT image by taking a product of the geometrical lens thickness (measured manually using the OCT calipers by adding the anterior and posterior thicknesses of the crystalline lens from the folded OCT image, $\tau_{a}+\tau_{b}$ ), and the refractive index used by the instrument (i.e. 1.43) (Figure 1c). The average refractive index of 1.43 for calculating geometrical distances in the mouse eye was chosen for the OCT values based on Schmucker and Schaeffel (2004b). Using the OCT, the value of $\delta$ was determined by measuring the change in position of the outer surface of the petri dish (below the crystalline lens), with that of the real position of the petri dish containing DMEM solution $\left(\delta_{\mathrm{b}}-\delta_{\mathrm{a}}\right.$, Figure 1c). The refractive index of DMEM $\left(n_{\mathrm{o}}\right)$ was found to be 1.3376 using an Abbe-3L refractometer (Milton Roy Co, Rochester, NY, USA).

The refractive index of the isolated lens $\left(n_{\mathrm{g}}\right)$ was then be calculated by dividing the optical thickness $(\tau)$ with the geometrical thickness $(t)$ of the lens.

$$
n_{\mathrm{g}}=\tau / t
$$

\subsection{SD-OCT validation for measuring the ocular lens refractive index in mice}

In order to examine the precision of our OCT system for calculating the lens refractive index in mice ex vivo, refractive index measurements were obtained for 5 standard ball lenses (each $2.00 \mathrm{~mm}$ diameter) of known refractive index (Edmund Optics Inc. Barrington, NJ, USA); fused silica $(n=1.458)$, N-BK-7 $(n=1.517)$, ruby $(n=1.77), N-L a S F 9(1.850)$ and high index lens (2.003) using OCT scans and the Uhlhorn's equation (1 and 2), as described above. Five rectangular scans $(4 \mathrm{~mm} \times 4 \mathrm{~mm}$ ) were used for calculating the average refractive index of the ball lenses. Ball lens materials were chosen to closely represent the previously reported range of lens refractive index in the mouse eye (Remtulla and Hallett, 1985; Schmucker and Schaeffel, 2004a).

\subsection{Normal refractive development}

To examine the changes in lens refractive index during normal visual input to the eye, the OCT measurements were performed on isolated crystalline lenses obtained from three different cohorts of mice, aged 4 (WT controls $n=10$; nob mice $n=10$ ), 10 (WT controls $\mathrm{n}=10 ; n o b$ mice $\mathrm{n}=11$ ) and 16 (WT controls $\mathrm{n}=10 ; n o b$ mice $\mathrm{n}=10$ ) weeks, respectively. In 
vivo OCT measurements were performed for all mice before sacrificing them at different ages.

\subsection{Form deprivation}

Disruption of form-vision through diffusers has been found to be associated with the development of myopia in mice (Barathi et al., 2008; Pardue et al., 2008; Qian et al., 2009; Schaeffel et al., 2004; Tejedor and de la Villa, 2003). Form deprivation was induced by placing a head-mounted goggling apparatus over the right eye at 4 weeks of age, as previously described (Faulkner et al., 2006). Briefly, a head pedestal attached to the skull held a small metal frame over the right eye. A plano lens painted with white nail polish was attached to the metal frame to be used as a diffuser. For form-deprivation experiments, 10 WT C57BL/6J (goggled $\mathrm{n}=5$; controls $\mathrm{n}=5$ ) and 11 nob mice (goggled $\mathrm{n}=5$; controls $\mathrm{n}=6$ ) had baseline refractions (using an eccentric infrared photorefractor, Schaeffel et al., 2004) and OCT at 4 weeks of age and then were form deprived for 4 weeks. At 8 weeks, refractive error and OCT measurements were repeated, and then ocular lenses were removed for refractive index measurements. Between week 4 and week 8 , the goggles were removed once a week (in dark) to allow the mice to clean their eyes.

\subsection{Data analysis}

In order to examine the precision and agreement for measurements on standard ball lenses and their known refractive indices, collected data were organized into agreement and BlandAltman plots (Altman and Bland, 1983). Bland-Altman coefficient of repeatability (CR) and intra-class correlation coefficient (ICC) indices were analyzed for precision and validity of the measurements, respectively. Differences in lens refractive index and lens thickness between WT and nob mice for different visual conditions were analyzed by two-way analysis of variance (ANOVA) and Holm-Sidak multiple comparisons using SigmaStat 3.5 (Systat Software, Inc., Chicago, IL). A linear regression analysis was performed to examine any significant association between the changes in lens refractive index and changes in ocular refraction of the mouse eye.

\section{Results}

\subsection{Agreement and precision of measurements on standard ball lenses}

Refractive index measurements on standard ball lenses and their known refractive indices provided by the manufacturer are summarized in Table 1 . There was a strong agreement between refractive index measurements performed using the OCT and manufacturer provided lens refractive index measures, with an average difference of only $\sim 0.05$ between the two measurements. This difference was due to a slight overestimation of geometrical lens thickness by the OCT (average difference $\sim 0.06 \mathrm{~mm}$ ) compared to manufacturer provided lens thickness of $2.00 \mathrm{~mm}$ for all standard lenses. A strong ICC and Bland-Altman CR indicated a good precision and validity for lens refractive index measurements with the OCT and their known refractive indices (ICC, 0.998 and CR, 0.116, Table 1). 


\subsection{Normal refractive development}

When measured at 4, 10, and 16 weeks of age under normal laboratory visual environments, both nob and WT mice showed a significant increase (expressed as the combined mean of both the right and left eyes) in geometrical crystalline lens thickness with age (two-way ANOVA main effect of age, $F(2,60)=178.4, p<0.001$, Figure 2). With increasing age, the average lens thickness (mean $\pm \mathrm{SD}$ ) increased from $1.78 \pm 0.03,2.02 \pm 0.05$ to $2.12 \pm 0.06$ $\mathrm{mm}$ for WT mice, and $1.79 \pm 0.08,2.01 \pm 0.04$ to $2.09 \pm 0.06 \mathrm{~mm}$ for nob mice at 4,10 and 16 weeks respectively. The lens thickness was not found to be significantly different between the two genotypes at any age (mean lens thickness for all ages; WT $1.97 \pm 0.18$ $\mathrm{mm}$, nob $1.96 \pm 0.16 \mathrm{~mm}$, two-way ANOVA main effect of strain $\mathrm{F}(1,60)=0.35, \mathrm{p}=0.557)$.

For normal unmanipulated visual development, refractive index for isolated crystalline lenses in nob mice was significantly greater than WT mice (mean for all ages WT: $1.42 \pm$ 0.01, nob: $1.44 \pm 0.001$; two-way ANOVA main effect of strain, $\mathrm{F}(1,60)=12.7, \mathrm{p}<0.001$ ). The refractive index differences between strains were largest at younger ages [mean for 4 (WT: $1.41 \pm 0.01, n o b: 1.44 \pm 0.03$ ) and 10 (WT: $1.42 \pm 0.02, n o b: 1.44 \pm 0.02$ ) weeks], with both reaching similar levels at later ages [mean refractive index at 16 weeks, $1.44 \pm$ 0.01 and $1.44 \pm 0.01$ for WT and nob mice respectively, p>0.05, Figure 2]. In addition, there was a strong trend for increasing refractive index in WT mice with age, but not the nob mice (two-way interaction $\mathrm{F}(2,60)=2.75, \mathrm{p}=0.07$, Figure 2).

\subsection{Form deprivation}

To examine whether visual environment affects the crystalline lens refractive index in mice, lens refractive index was compared between nob and WT mice after form deprivation. In addition to a significantly larger myopic shift (the difference between the goggled and opposite eye) in the nob mice (mean refraction $\pm \mathrm{SD} ;-3.76 \pm 0.96 \mathrm{D}$ ) compared to the WT mice $(-2.02 \pm 1.68 \mathrm{D})$ after 4 weeks of goggling (one-way ANOVA, $\mathrm{F}(1,10)=5.35$, $\mathrm{p}=0.046$ ), both genotypes were confirmed to have a significantly greater myopic refractive shift than their respective controls (mean refraction; WT, $0.05 \pm 0.99$ and nob, $-0.44 \pm 0.62$ D, two-way ANOVA, $\mathrm{F}(1,18)=20.81, \mathrm{p}<0.001)$. Additionally, no significant differences in body weight were found between the two genotypes either at the baseline (4 weeks; mean body weight, WT $14.41 \pm 1.36 \mathrm{~g}$, nob $14.15 \pm 1.83 \mathrm{~g}$ ), or after 4 weeks of form-deprivation treatment (WT $23.41 \pm 1.92 \mathrm{~g}$, nob $23.15 \pm 2.17 \mathrm{~g}$ ) (two-way ANOVA, $\mathrm{F}(1,41)=0.123$, $\mathrm{p}=0.73$ ), suggesting that changes in the crystalline lens associated with goggling were not confounded by differences in body size between the two genotypes.

In response to form deprivation, a thinning of the crystalline lens was observed compared to naïve controls of both strains that reached borderline statistical significance (geometrical lens thickness; control> opposite eye> goggled eye, two-way ANOVA for main effect of treatment, $\mathrm{F}(2,39)=3.2, \mathrm{p}=0.052$, Table 2 ). The crystalline lens thickness in response to goggling was not significantly different between the two mouse strains.

For both strains, no significant differences in the lens refractive index between the goggled and opposite eyes ( $>0.05$, Table 2 ) were measured. However, refractive index was significantly different between treatment groups in $n o b$ mice, while not showing any 
differences in WT mice (two-way ANOVA interaction effect, $\mathrm{F}(2,39)=5.45, \mathrm{p}=0.009$; Figure 3). Both eyes of form deprived nob mice had significantly greater crystalline lens refractive index (goggled, $1.49 \pm 0.01$; opposite $1.47 \pm 0.03$ ) compared to their naïve controls (expressed as the average from both the right and the left eye; mean, $1.45 \pm 0.02$, Table 2, Holm-Sidak multiple comparisons, $\mathrm{p}<0.05)$. In contrast, when diffuser goggles were applied to the WT mice, no significant shift of lens refractive index was detected between different treatment groups (googled eyes mean $1.44 \pm 0.01$; opposite eyes mean $1.43 \pm 0.01$; control eyes mean $1.44 \pm 0.01, \mathrm{p}>0.05$, Figure 3 ). Additionally, as expected from Figure 3, the refractive index of nob and WT lenses were significantly different (twoway ANOVA main effect of strain $F(1,39)=43.4$, p $<0.001$, Figure 3$)$. No significant association was found between the changes in lens refractive index (the difference between the right and the left eye) and refractive shift observed in the goggled animals (slope $=$ $-0.0004, \mathrm{r}^{2}=0.03, \mathrm{p}=0.871$ ).

\subsection{Comparison of different methods for measuring the lens refractive index}

In order to examine the reliability of lens refractive index values measured in our study for the calculation of lens thickness in mice, we compared the geometrical lens thickness measurements from the OCT (in vivo using a refractive index of 1.43, LT OCT) to lens thickness measurements recalculated using the refractive index values from Schmucker and Schaeffel (2004a, LT Sch), individual isolated crystalline lenses measured in this study (LT Exp), and lens thicknesses calculated from the average refractive index of each age group (LT Avg Age) (Figure 4). Refractive index values obtained from each method showed a significant increase $(p<0.001)$ in the crystalline lens thickness with age for WT mice, measured at 4, 10 and 16 weeks of age. The geometrical lens thickness measured from LT OCT was not significantly different from lens thicknesses calculated from LT Exp or LT Avg Age. Additionally, we found a strong correlation between the individual geometrical lens thicknesses measured in vivo (LT OCT) and in vitro (LT Exp) using the OCT (slope = 0.9633, $\left.\mathrm{r}^{2}=0.99, \mathrm{p}<0.001\right)$. The lens thickness estimated using LTSch from the regression analysis reported by Schmucker and Schaeffel (2004a) exhibited $~ 10 \%$ lower values (Holm-Sidak multiple comparisons, $\mathrm{p}<0.05$ ) for all ages compared to other methods (Figure 4).

\section{Discussion}

Recently, there has been interest in establishing the mouse as a model for studying refractive development and myopia. In mice, the crystalline lens is the most important optical component (Pardue et al., 2013; Schmucker and Schaeffel, 2004a), occupying a large proportion of the entire globe. Knowing the profile of lens thickness and refractive index in the mouse eye is important for accurately interpreting the results from optical interferometry techniques (such as PCI and OCT), a key instrument for evaluating ocular parameters in this small eye (Pardue et al., 2013; Park et al., 2012) that requires refractive index values for calculating a geometric length. In this study, we investigated the changes in lens thickness and refractive index of two different mouse strains (WT and nob mice) under unmanipulated and form deprived visual conditions. We have shown an increase in the crystalline lens thickness for both strains and a strong trend for increase in refractive index only for WT 
mice with age under unmanipulated visual conditions. With form-deprivation, we observed a strong trend for thinning of the ocular lens and significant increase in lens refractive index for both eyes of goggled nob mice compared to naïve controls.

\subsection{SD-OCT for measuring the lens refractive index in mice}

Non-destructive methods of measuring lens refractive index using measurements of angular deviation of laser beam passed through the lens (Campbell., 1984), optic fiber probe (Pierscionek, 1997), scheimpflug images (Dubbelman and Van der Heijde, 2001), and most recently the magnetic resonance imaging (MRI, Jones et al., 2005, 2007) have replaced older destructive techniques that caused artifacts due to sectioning and storage of frozen tissues. In the present study, we used a novel non-destructive technique for measuring the lens refractive index in the mouse eye, by imaging isolated crystalline lenses using an OCT system as described by Uhlhorn et al (2008). Previously, the OCT was reliably used to examine the refractive index of the crystalline lens in the zebrafish (Rao et al., 2006). An ICC and CR of 0.998 and 0.116 respectively between the lens refractive index measurements performed on standard ball lenses and their known refractive indices demonstrate the reliability for using the OCT for the calculation of lens refractive index in rodent eyes.

\subsection{Crystalline lens refractive index in the mouse eye}

Previous studies examining the lens refractive index in rodents have used paraxial ray tracing on radii of curvature and axial distances obtained from frozen sections (Remtulla and Hallett, 1985), or frozen eyes bisected horizontally (Schmucker and Schaeffel., 2004a). The equivalent lens refractive index values from these two studies were considerably higher than biologically possible limits of the eye (i.e. <1.55, Hughes, 1979). Hughes (1979) using the two shell lens model (von Helmholtz, 1909) that closely approximates the living eye due to a reasonably smaller separation between the two principal points of the lens, calculated the refractive index of the cortex and the core of the crystalline lens in rat eyes to be 1.390 and 1.500 respectively in order to produce emmetropic to low hyperopic refractions in rodents. Conversely, in later studies, the average refractive index for a C57BL/6 mouse 14 (Schmucker and Schaeffel., 2004a) to 20 (Remtulla and Hallett, 1985) weeks-old was calculated to be 1.605 and 1.659 respectively. In the current study, the lens refractive index was found to be substantially lower than previously reported values on murine eyes (average refractive index for 16 weeks old mice, 1.44). In the study by Remtulla (1985), sectioning artifacts, insufficient resolution from frozen sections, tissue storage, and instruments used for measuring the radii of curvature may have influenced the values of lens refractive index (Pardue et al., 2013). On the other hand, Schmucker and Schaeffel (2004a) used refractive indices of the optical media from Remtulla and Hallett (1985), and performed a paraxial ray tracing of frozen eyes hemisected with a microtome to determine the refractive index of murine crystalline lens and would also be subject to freezing artifacts. Unlike previous studies, our method of imaging the isolated crystalline lens provided a non-destructive, direct measure of refractive index independent of any schematic eye modelling from other ocular components. Although using different techniques, our measurements of lens refractive index are comparable with previous reports of equivalent lens refractive index in other rodents (Massof and Chang, 1972; Phillipson, 1969). In the present study, the index of 
refraction was measured only at the optical axis of the lens, and the crystalline lens was represented as an optical medium of homogenous refractive index (Massof and Chang, 1972). This may not necessarily reflect the true refractive index of the tissue because the crystalline lens in murine eyes follow a gradient of refractive index (Campbell and Hughes, 1981; Phillipson, 1969), producing variations in optical power of the lens throughout its surface, which has been shown to be essential for predicting the optimum refractive state in rodent eyes (Campbell and Hughes, 1981). Therefore, an increase or decrease in the refractive index of the crystalline lens may alternatively represent changes in the refractive index gradient profile of the lens. Future studies examining the structure of the gradient lens index would allow better comparison with other reports of gradient lens refractive index in rodents (Campbell and Hughes, 1981; Hughes, 1979).

\subsection{Lens thickness and refractive index during normal refractive development}

For both WT and nob mice with normal refractive development, we observed a significant increase in lens thickness from 4 to 16 weeks of age. Previous studies have also reported an increase in lens thickness of C57BL/6 mice with age using various techniques, such as optical interferometry (Puk et al., 2006), frozen sections (Schmucker and Schaeffel, 2004a), OCT (Chou et al., 2011; Zhou et al., 2008) and MRI (Tkatchenko et al., 2010a). The mean increase in lens thickness of $\sim 0.3 \mathrm{~mm}$ during 12 weeks of measurement period was consistent with previous reports on rodent eyes (Puk et al., 2006; Schmucker and Schaeffel, 2004a; Tkatchenko et al., 2010a).

For unmanipulated visual development, we found a strong trend for increase in the lens refractive index for the WT C57BL/6 mice with age. An increase in refractive index of the crystalline lens with age has been previously reported in C57BL/6 mice (Schmucker and Schaeffel, 2004a), as well as in other animal species (Pierscionek, 1989). However, we didn't find any significant age-related changes in the lens refractive index of nob mice. If the lens refractive index was an important determinant of refractive error in C57BL/6J mice, an increase in refractive index of the crystalline lens during ocular development would move the focal point of the eye towards myopia. As a paradox, most studies have found the WT C57BL/6J mice to have hyperopic refractions that become relatively more hyperopic until approximately 6 - 8 weeks of age before they stabilize (Pardue et al., 2008; Schaeffel et al., 2004; Schmucker and Schaeffel, 2004a; Yu et al., 2011; Zhou et al., 2008, 2010).

Additionally, Pardue et al (2008) reported an increase in hyperopic refraction of nob mice until about 6 weeks of age followed by a relative myopic shift. Despite a significant increase in axial length (Schmucker and Schaeffel, 2004a; Zhou et al., 2008) and small changes in lens refractive index both causing a myopic refractive shift, the paradox of hyperopic refractions in mice could potentially be explained by variations in other lenticular components (such as lens curvature, thickness or displacement of the lens) during normal eye growth. Previous studies have reported a significant positive correlation between the changes in lens thickness and refractive error in mice (Tkatchenko et al., 2010a; Zhou et al., 2008). 


\subsection{Inter-strain differences in lens refractive index}

At younger ages (4 and 10 weeks of age), the lens refractive index of nob mice was significantly greater than WT mice. Despite the higher refractive index, nob mice have been reported to have significantly more hyperopic refractions at young ages than the WT mice ( 2 D greater at 4 weeks; Pardue et al., 2008), further indicating that the crystalline lens refractive index may not be the most important predictor for ocular refraction in mice. Differences in refractive index between strains could be associated with differences in lens protein content (Pickett-Seltner et al., 1987) or distribution of lens fibers in the crystalline lens. Inter-species differences in the crystalline lens refractive index have been previously reported in fish eyes (Sadler, 1973).

\subsection{Lens refractive index during form-deprivation}

Another important finding from this study is a significant myopic shift, and an increase in refractive index of the crystalline lens with form-deprivation treatment in nob mice. The absence of ON pathway function leading to low dopaminergic activity in the nob mouse has previously been associated with an increased susceptibility to myopia in response to visual form-deprivation (Pardue et al., 2008). It is known that nuclear cataract causes an increase in lens refractive index and negative spherical aberration (Kuroda et al., 2002), leading to a significant myopic shift in human eyes (Brown and Hill, 1987; Pesudovs and Elliott, 2003), suggesting that the changes in lens refractive index could potentially influence the susceptibility for myopic refractive error. We observed no changes in ocular clarity between the nob and WT mice. In our study with form-deprivation, although the lens refractive index was found to be significantly greater in nob mice compared to WT, no significant association was observed between the changes in lens refractive index and the magnitude of myopic refractions associated with goggling. Previously, changes in the crystalline lens curvature (McBrien et al., 1995) and lens protein (Pickett-Seltner et al., 1987) have been associated with experimentally induced myopia in chicks. Further studies examining the changes in the crystalline lens curvature or protein contents with visual deprivation in different mouse strains are required to better understand the crystalline lens changes and its association with myopia development in murine eyes.

\subsection{Lens refractive index measurements for calculating the lens thickness in mice}

Finally, geometrical lens thickness measurements from the OCT for normally developed WT mice (in vivo using a refractive index of 1.43) were recalculated using the refractive index values from Schmucker and Schaeffel (2004a), isolated crystalline lenses measured in this study, and the average refractive index of isolated lenses for each age group (Figure 4). The geometrical lens thickness measured from the OCT in vivo were found to be similar to lens thicknesses calculated from the individual isolated crystalline lenses measured in this study (with a strong positive correlation between the two, slope $=0.9633, \mathrm{p}<0.001$ ), as well as lens thicknesses calculated from the average refractive index of each age group. The geometrical lens thickness estimated using refractive index from the regression analysis shown by Schmucker and Schaeffel (2004a) showed significantly lower values compared to other methods. It is to be noted that the method used by Schmucker and Schaeffel (indirectly calculated from paraxial ray tracing) was different from our direct method of calculating the 
crystalline lens refractive index in the mouse eye. The results from this analysis suggest that the representative average refractive index for each age group calculated in our study can be effectively used for calculating the lens thickness in mice.

The mean lens refractive index found in our study (mean refractive index, 1.41, 1.42 and 1.44 for 4,10 and 16 weeks of age) was proximal to the index of refraction used by the SDOCT (i.e. 1.43, Schmucker and Schaeffel, 2004b) for calculating geometrical distances within the mouse eye. Research applications requiring precise optical measurements in rodents should take these lens refractive indices into account when interpreting the OCT data. However, caution should be exercised in using these results for different mouse strains not measured in this study.

In conclusion, we have shown a novel, non-destructive and direct method of measuring the crystalline lens refractive index in the mouse eye in vitro. The lens thickness increases significantly with age during normal unmanipulated visual development in mice. At younger ages, the lens refractive index of nob mice is significantly greater than WT mice, suggesting inter-strain differences in lens refractive index of normal visually developing mice. Formdeprivation causes a significant increase in lens refractive index for both eyes in goggled nob mice.

\section{Acknowledgments}

We would like to acknowledge Dr. Adrian Glasser, University of Houston, for insightful discussions on this topic and assistance with optical modeling of the mouse eye.

\section{References}

Altman DG, Bland JM. Measurement in medicine: the analysis of method comparison studies. Statistician. 1983; 32:307-317.

Augusteyn RC, Rosen AM, Borja D, Ziebarth NM, Parel JM. Biometry of primate lenses during immersion in preservation media. Mol. Vis. 2006; 12:740-747. [PubMed: 16865087]

Barathi VA, Boopathi VG, Yap EP, Beuerman RW. Two models of experimental myopia in the mouse. Vision. Res. 2008; 48:904-916. [PubMed: 18289630]

Brown NA, Hill AR. Cataract: the relation between myopia and cataract morphology. Br. J. Ophthalmol. 1987; 71:405-414. [PubMed: 3620419]

Campbell MC, Hughes A. An analytic, gradient index schematic lens and eye for the rat which predicts aberrations for finite pupils. Vision. Res. 1981; 21:1129-1148. [PubMed: 7314492]

Campbell MC. Measurement of refractive index in an intact crystalline lens. Vision. Res. 1984; 24:409-415. [PubMed: 6740962]

Chou TH, Kocaoglu OP, Borja D, Ruggeri M, Uhlhorn SR, Manns F, Porciatti V. Postnatal elongation of eye size in DBA/2J mice compared with C57BL/6J mice: in vivo analysis with whole-eye OCT. Invest. Ophthalmol. Vis. Sci. 2011; 52:3604-3612. [PubMed: 21372015]

Crewther DP. The role of photoreceptors in the control of refractive state. Prog. Retin. Eye. Res. 2000; 19:421-457. [PubMed: 10785617]

Dubbelman M, Van der Heijde GL. The shape of the aging human lens: curvature, equivalent refractive index and the lens paradox. Vision. Res. 2001; 41:1867-1877. [PubMed: 11369049]

Edwards MH. Animal models of myopia. A review. Acta. Ophthalmol. Scand. 1996; 74:213-219. [PubMed: 8828713]

Faulkner AE, Kim MK, Iuvone PM, Pardue MT. Head-mounted goggles for murine form deprivation myopia. J. Neurosci. Methods. 2007; 161:96-100. [PubMed: 17126909] 
Garner LF, Yap M, Smith G. Crystalline lens dimensions and refractive index in schoolchildren. Optom. Vis. Sci. 1994; 71:150. [AAO Annual Meeting Abstracts].

Gordon RA, Donzis PB. Refarctive development of the human eye. Arch. Ophthalmol. 1985; 103:785789. [PubMed: 4004614]

Gregg RG, Mukhopadhyay S, Candille SI, Ball SL, Pardue MT, McCall MA, Peachey NS. Identification of the gene and the mutation responsible for the mouse nob phenotype. Invest. Ophthalmol. Vis. Sci. 2003; 44:378-384. [PubMed: 12506099]

Hughes A. A schematic eye for the rat. Vision. Res. 1979; 19:569-588. [PubMed: 483586]

Hung L-F, Crawford MLJ, Smith EL. Spectacle lenses alter eye growth and refractive status of young monkeys. Nature. Med. 1995; 1:761-765. [PubMed: 7585177]

Jiang M, Wu PC, Fini ME, Tsai CL, Itakura T, Zhang X, Jiao S. Single-shot dimension measurements of the mouse eye using SD-OCT. Ophthalmic. Surg. Lasers. Imaging. 2012; 43:252-256. [PubMed: 22421200]

Jones CE, Atchison DA, Meder R, Pope JM. Refractive index distribution and optical properties of the isolated human lens measured using magnetic resonance imaging (MRI). Vision. Res. 2005; 45:2352-2366. [PubMed: 15979462]

Jones CE, Atchison DA, Pope JM. Changes in lens dimensions and refractive index with age and accommodation. Optom. Vis. Sci. 2007; 84:990-995. [PubMed: 18049365]

Kuroda T, Fujikado T, Maeda N, Oshika T, Hirohara Y, Mihashi T. Wavefront analysis in eyes with nuclear or cortical cataract. Am. J. Ophthalmol. 2002; 134:1-9. [PubMed: 12095801]

Massof RW, Chang FW. A revision of the rat schematic eye. Vision. Res. 1972; 12:793-796. [PubMed: 5037702]

McBrien NA, Moghaddam HO, Cottriall CL, Leech EM, Cornell LM. The effects of blockade of retinal cell action potentials on ocular growth, emmetropization and form deprivation myopia in young chicks. Vision. Res. 1995; 35:1141-1152. [PubMed: 7610575]

Mutti DO, Zadnik K, Adams AJ. A revised equivalent refractive index of the crystalline lens based on in vivo measurements from the Orinda Longitudinal Study of Myopia. Invest. Ophthalmol. Vis. Sci. 1991; 32:201. [ARVO Abstracts].

Mutti DO, Zadnik K, Fusaro RE, Friedman NE, Sholtz RI, Adams AJ. Optical and structural development of the crystalline lens in childhood. Invest. Ophthalmol. Vis. Sci. 1998; 39:120-133. [PubMed: 9430553]

Mutti DO, Mitchell GL, Jones LA, Friedman NE, Frane SL, Lin WK, Moeschberger ML, Zadnik K. Axial growth and changes in lenticular and corneal power during emmetropization in infants. Invest. Ophthalmol. Vis. Sci. 2005; 46:3074-3080. [PubMed: 16123404]

Norton TT, McBrien NA. Normal development of refractive state and ocular component dimensions in the tree shrew. Vision. Res. 1992; 32:833-842. [PubMed: 1604852]

Pardue MT, McCall MA, LaVail MM, Gregg RG, Peachey NS. A naturally occurring mouse model of X-linked congenital stationary night blindness. Invest. Ophthalmol. Vis. Sci. 1998; 39:2443-2449. [PubMed: 9804152]

Pardue MT, Faulkner AE, Fernandes A, Yin H, Schaeffel F, Williams RW, Pozdeyev N, Iuvone PM. High susceptibility to experimental myopia in a mouse model with a retinal on pathway defect. Invest. Ophthalmol. Vis. Sci. 2008; 49:706-712. [PubMed: 18235018]

Pardue MT, Stone RA, Iuvone PM. Investigating mechanisms of myopia in mice. Exp. Eye. Res. 2013; 114:96-105. [PubMed: 23305908]

Park, Hn; Qazi, Y.; Tan, C.; Jabbar, SB.; Cao, Y.; Schmid, G.; Pardue, MT. Assessment of axial length measurements in mouse eyes. Optom. Vis. Sci. 2012; 89:296-303. [PubMed: 22246334]

Pesudovs K, Elliott DB. Refractive error changes in cortical, nuclear, and posterior subcapsular cataracts. Br. J. Ophthalmol. 2003; 87:964-967. [PubMed: 12881335]

Phillipson B. Distribution of protein within the normal rat lens. Invest. Ophthalmol. 1969; 8:258-270. [PubMed: 5772717]

Pickett-Seltner RL, Weerheim J, Sivak JG, Pasternak J. Experimentally-induced myopia does not affect post-hatching development of the chick lens. Vision. Res. 1987; 27:1779-1782. [PubMed: 3445467]

Exp Eye Res. Author manuscript; available in PMC 2015 August 01. 
Pierscionek BK. Growth and ageing effects on the refractive index in the equatorial plane of the bovine lens. Vision. Res. 1989; 29:1759-1766. [PubMed: 2631397]

Pierscionek BK. Refractive index contours in the human lens. Exp. Eye. Res. 1997; 64:887-893. [PubMed: 9301469]

Puk O, Dalke C, Favor J, de Angelis MH, Graw J. Variations of eye size parameters among different strains of mice. Mamm. Genome. 2006; 17:851-857. [PubMed: 16897341]

Qian YS, Chu RY, Hu M, Hoffman MR. Sonic hedgehog expression and its role in form-deprivation myopia in mice. Curr. Eye. Res. 2009; 34:623-635. [PubMed: 19899989]

Rafferty NS, Goossens W. Cytoplasmic filaments in the crystalline lens of various species: functional correlations. Exp. Eye. Res. 1978; 26:177-190. [PubMed: 305361]

Rao KD, Verma Y, Patel HS, Gupta PK. Non-invasive ophthalmic imaging of adult zebrafish eye using optical coherence tomography. Curr. Sci. India. 2006; 90:1506-1510.

Remtulla S, Hallett PE. A schematic eye for the mouse, and comparisons with the rat. Vision. Res. 1985; 25:21-31. [PubMed: 3984214]

Sadler JD. The focal length of the fish eye lens and visual acuity. Vision. Res. 1973; 13:417-423. [PubMed: 4692529]

Schaeffel F, Glasser A, Howland HC. Accommodation, refractive error and eye growth in chickens. Vision. Res. 1988; 28:639-657. [PubMed: 3195068]

Schaeffel F, Burkhardt E, Howland HC, Williams RW. Measurement of refractive state and deprivation myopia in two strains of mice. Optom. Vis. Sci. 2004; 81:99-110. [PubMed: 15127929]

Schaeffel, F. The mouse eye as a model for myopia, and optics of the eye. In: Chalupa, LM.; Williams, RW., editors. Eye, Retina and Visual System of the Mouse. Cambridge: The MIT Press; 2008. p. 73-85.

Schmitt JM. Optical coherence tomography (OCT): a review. IEEE. 1999; 5:1205-1215.

Schmucker C, Schaeffel F. A paraxial schematic eye model for the growing C57BL/6 mouse. Vision. Res. 2004a; 44:1857-1867. [PubMed: 15145680]

Schmucker C, Schaeffel F. In vivo biometry in the mouse eye with low coherence interferometry. Vision. Res. 2004b; 44:2445-2456. [PubMed: 15358080]

Shen W, Vijayan M, Sivak JG. Inducing form-deprivation myopia in fish. Invest. Ophthalmol. Vis. Sci. 2005; 46:1797-1803. [PubMed: 15851585]

Siegwart JT Jr, Norton TT. Perspective: how might emmetropization and genetic factors produce myopia in normal eyes? Optom. Vis. Sci. 2011; 8:E365-E372. [PubMed: 21258261]

Smith EL III, Hung L-F. Form-deprivation myopia in monkeys is a graded phenomenon. Vision. Res. 2000; 40:371-381. [PubMed: 10820617]

Tejedor J, de la Villa P. Refractive changes induced by form deprivation in the mouse eye. Invest. Ophthalmol. Vis. Sci. 2003; 44:32-36. [PubMed: 12506052]

Tkatchenko TV, Shen Y, Tkatchenko AV. Analysis of postnatal eye development in the mouse with high-resolution small animal magnetic resonance imaging. Invest. Ophthalmol. Vis. Sci. 2010a; 51:21-27. [PubMed: 19661239]

Tkatchenko TV, Shen Y, Tkatchenko AV. Mouse experimental myopia has features of primate myopia. Invest. Ophthalmol. Vis. Sci. 2010b; 51:1297-1303. [PubMed: 19875658]

Troilo D, Judge SJ. Ocular development and visual deprivation myopia in the common marmoset (Callithrix jacchus). Vision. Res. 1993; 33:1311-1324. [PubMed: 8333155]

Uhlhorn SR, Borja D, Manns F, Parel JM. Refractive index measurement of the isolated crystalline lens using optical coherence tomography. Vision. Res. 2008; 48:2732-2738. [PubMed: 18824191]

von Helmholtz, H. Handbuch der physiologischen Optik. In: Gullstrand, et al., editors; Southall, JPC., translator. Optical Society of America. third ed.. 1909. English translation edited by (1924).

Wallman J, Adams JI. Developmental aspects of experimental myopia in chicks: susceptibility, recovery and relation to emmetropization. Vision. Res. 1987; 27:1139-1163. [PubMed: 3660666]

Wallman J, Wildsoet C, Xu A, Gottlieb MD, Nickla DL, Marran L, Krebs W, Christensen AM. Moving the retina: choroidal modulation of refractive state. Vision. Res. 1995; 35:37-50. [PubMed: 7839608] 
Wallman J, Winawer J. Homeostasis of eye growth and the question of myopia. Neuron. 2004; 43:447-468. [PubMed: 15312645]

Wang L, Považay B, Chen YP, Hofer B, Drexler W, Guggenheim JA. Heritability of ocular component dimensions in mice phenotyped using depth-enhanced swept source optical coherence tomography. Exp. Eye. Res. 2011; 3:482-490. [PubMed: 21726551]

Wildsoet CF. Active emmetropization - evidence for its existence and ramifications for clinical practice. Ophthalmic. Physiol. Opt. 1997; 17:279-290. [PubMed: 9390372]

Yu Y, Chen H, Tuo J, Zhu Y. Effects of flickering light on refraction and changes in eye axial length of C57BL/6 mice. Ophthalmic. Res. 2011; 46:80-87. [PubMed: 21273796]

Zadnik K, Mutti DO, Fusaro RE, Adams AJ. Longitudinal evidence of crystalline lens thinning in children. Invest. Ophthalmol. Vis. Sci. 1995; 36:1581-1587. [PubMed: 7601639]

Zhou X, Qu J, Xie R, Wang R, Jiang L, Zhao H, Wen J, Lu F. Normal development of refractive state and ocular dimensions in guinea pigs. Vision. Res. 2006; 46:2815-2823. [PubMed: 16723148]

Zhou X, Shen M, Xie J, Wang J, Jiang L, Pan M, Qu J, Lu F. The development of the refractive status and ocular growth in C57BL/6 mice. Invest. Ophthalmol. Vis. Sci. 2008; 49:5208-5214. [PubMed: 18689702]

Zhou X, An J, Wu X, Lu R, Huang Q, Xie R, Jiang L, Qu J. Relative axial myopia induced by prolonged light exposure in C57BL/6 mice. Photochem. Photobiol. 2010; 86:131-137. [PubMed: 19912561] 


\section{Highlights}

Optical coherence tomography can be used to measure mouse lens refractive index.

Mouse crystalline lens thickness increased with age.

Mouse lens refractive index is altered by the retinal gene mutation $N y x$ in nob mice.

Mouse lens refractive index increased with form deprivation myopia in nob mice. 

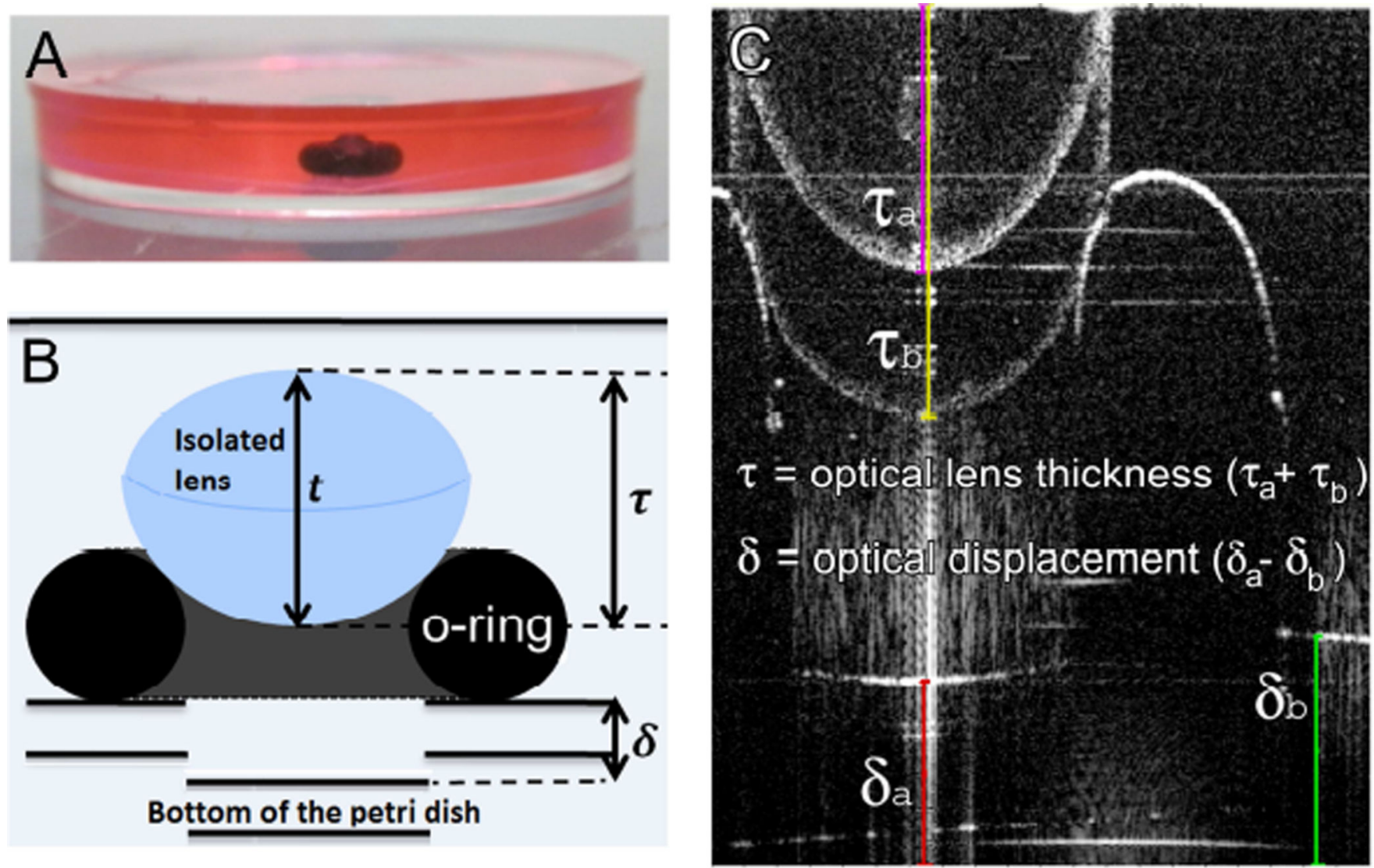

Figure 1.

Measurement of the crystalline lens refractive index in the mouse eye. (A) The lens measurement chamber, with the isolated mouse lens placed inside a plastic petri dish filled with DMEM solution. (B) Measurement of geometrical lens thickness $(t)$ using the optical lens thickness $(\tau)$, optical displacement $(\delta)$ and refractive index of the DMEM medium $\left(n_{\mathrm{o}}\right)$ for the calculation of lens refractive index in mice. (C) A typical OCT scan for an isolated mouse lens showing measurements of optical lens thickness $\left[\tau,\left(\tau_{a}+\tau_{b}\right)=1.43\right]$ where $\tau_{a}$ and $\tau_{b}$ are the anterior and posterior thicknesses of the crystalline lens respectively from the folded OCT image, and optical displacement $\left(\delta, \delta_{\mathrm{b}}-\delta_{\mathrm{a}}\right)$ where $\delta_{\mathrm{a}}$ is the change in position of the outer surface of the petri dish (below the crystalline lens) and $\delta_{\mathrm{b}}$ is the real position of the petri dish containing DMEM solution. 

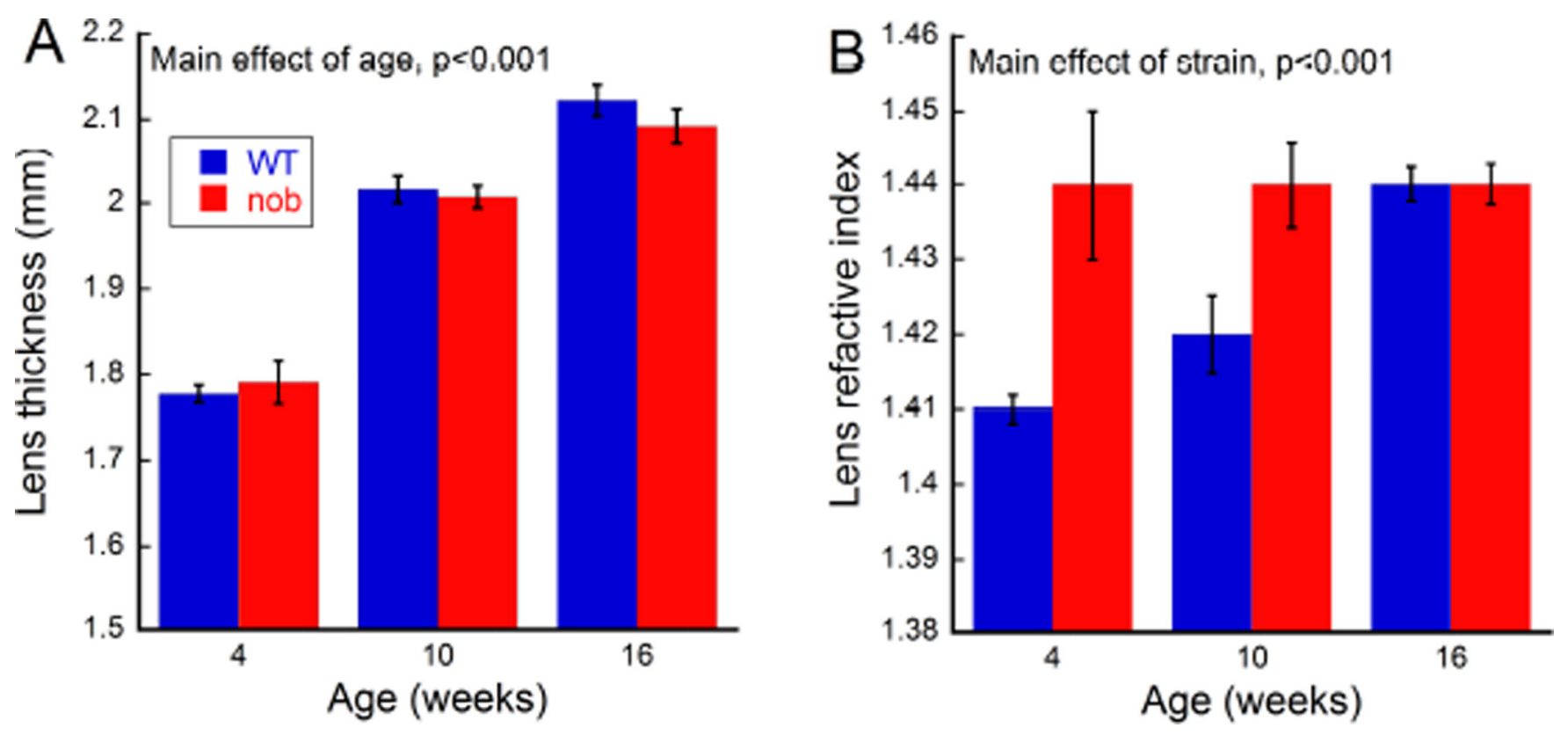

Figure 2.

Lens thickness (A) and refractive index (B) from isolated crystalline lenses in the mouse eye measured at 4, 10, and 16 weeks of age. (A) Both nob and WT mice showed a significant increase in the crystalline lens thickness with age (two-way ANOVA main effect of age, $\mathrm{F}(2,60)=178.4, \mathrm{p}<0.001)$. (B) Lens refractive index in nob mice was significantly greater compared to the WT mouse (two-way ANOVA, main effect for strain, $\mathrm{F}(1,60)=12.7$, $\mathrm{p}<0.001)$. Data are expressed as the mean \pm SEM. 

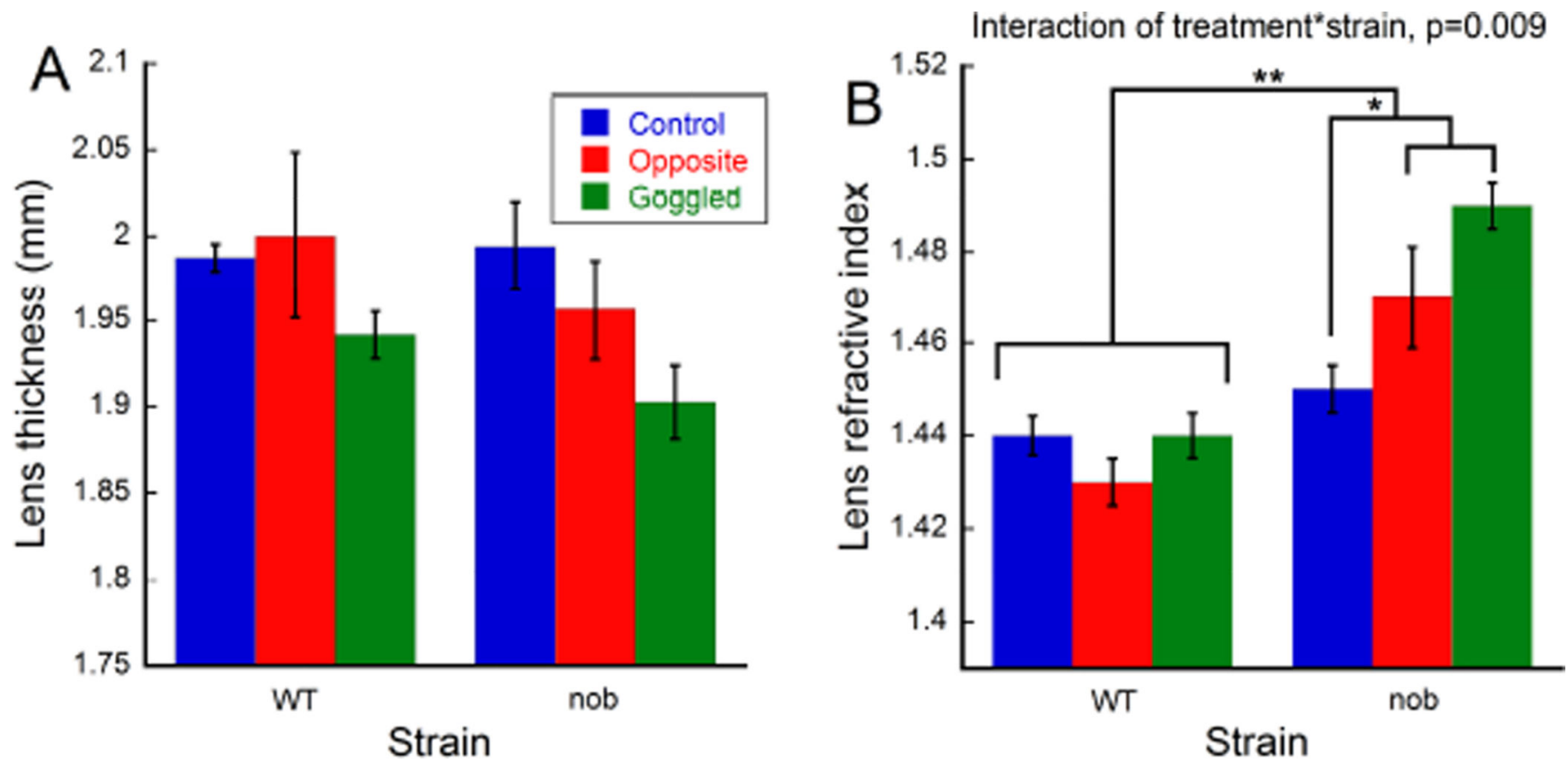

Figure 3.

Lens thickness (A) and refractive index (B) measurements from the eyes of WT and nob eyes from the three treatment groups: form deprivation induced by diffuser goggles, the unmanipulated opposite eye and naïve control mice. (A) Following 4 weeks of visual deprivation, both strains showed a strong trend for thinning of the crystalline lens associated with goggling compared to their naïve controls (two-way $\operatorname{ANOVA,~} F(2,39)=3.2, p=0.052$ ). (B) The nob mice had significantly greater lens refractive index compared to WT mice (twoway ANOVA main effect of strain $\mathrm{F}(1,39)=43.4$, $\mathrm{p}<0.001)$. Lens refractive index was significantly different between treatment groups, dependent on the strain (two-way ANOVA interaction effect, $\mathrm{F}(2,39)=5.45, \mathrm{p}=0.009)$. Refractive index measured after 4 weeks of form-deprivation showed a significantly greater refractive index in the goggled and opposite eyes of nob mice compared to their naïve controls (Holm-Sidak multiple comparisons, $\mathrm{p}<0.05)$. The asterisk signs $*$ and $* *$ represent $\mathrm{p}<0.05$ and $\mathrm{p}<0.001$ respectively. Data are expressed as the mean \pm SEM. 


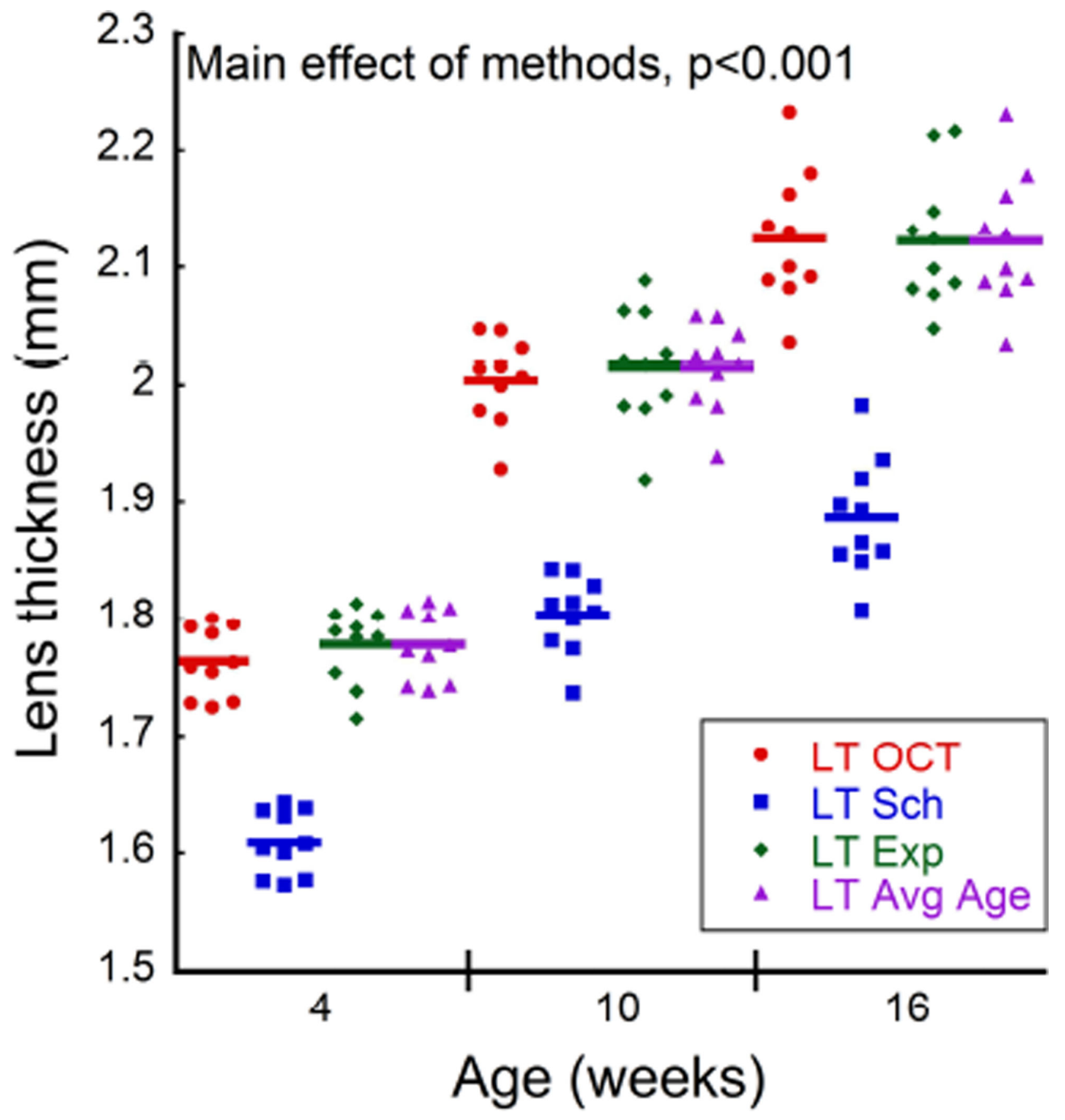

Figure 4.

Dot plot illustrating comparison of geometrical lens thicknesses measured using the refractive index values from the OCT (in vivo measurements using a refractive index of 1.43, LT OCT), regression analysis reported by Schmucker and Schaeffel (2004a, LT Sch), individual isolated crystalline lenses measured in the current study (LT Exp), and lens thicknesses calculated from the average refractive index of each age group (LT Avg Age). Data are expressed as the mean \pm SEM. 


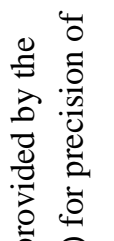

文

过

:

:

प्र

言

ब

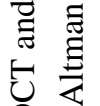

के

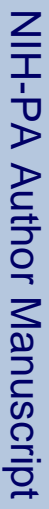

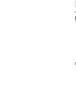

$\overbrace{0,0} \bar{\Xi}$

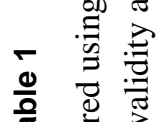

은

泀

疍

过

可

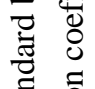

胥

苛

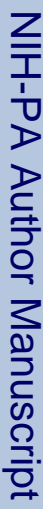

\begin{tabular}{|c|c|c|}
\hline$\underset{U}{U}$ & $\begin{array}{l}a \\
: \\
\square\end{array}$ & \\
\hline ปี & & \\
\hline 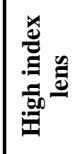 & 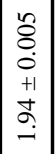 & ¿̊. \\
\hline 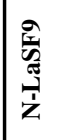 & $\begin{array}{c}\vec{\Xi} \\
\dot{0} \\
+1 \\
\dot{+1} \\
\dot{\infty} \\
-\end{array}$ & 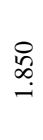 \\
\hline $\overrightarrow{\overrightarrow{\bar{z}}}$ & 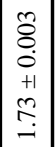 & $\stackrel{\text { F }}{\mathrm{I}}$ \\
\hline$\frac{\hat{y}}{\stackrel{y}{*}}$ & \begin{tabular}{c}
$\overrightarrow{8}$ \\
0 \\
0 \\
+1 \\
\multirow{f}{f}{} \\
-
\end{tabular} & $\underline{\underline{n}}$ \\
\hline 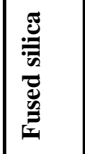 & $\begin{array}{c}0 \\
\delta \\
0 \\
0 \\
+1 \\
o \\
\dot{+} \\
-\end{array}$ & 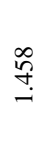 \\
\hline 惫 & $\begin{array}{l}\text { ப் } \\
\text { 1े } \\
\text { की }\end{array}$ & 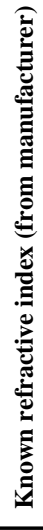 \\
\hline
\end{tabular}

Exp Eye Res. Author manuscript; available in PMC 2015 August 01. 


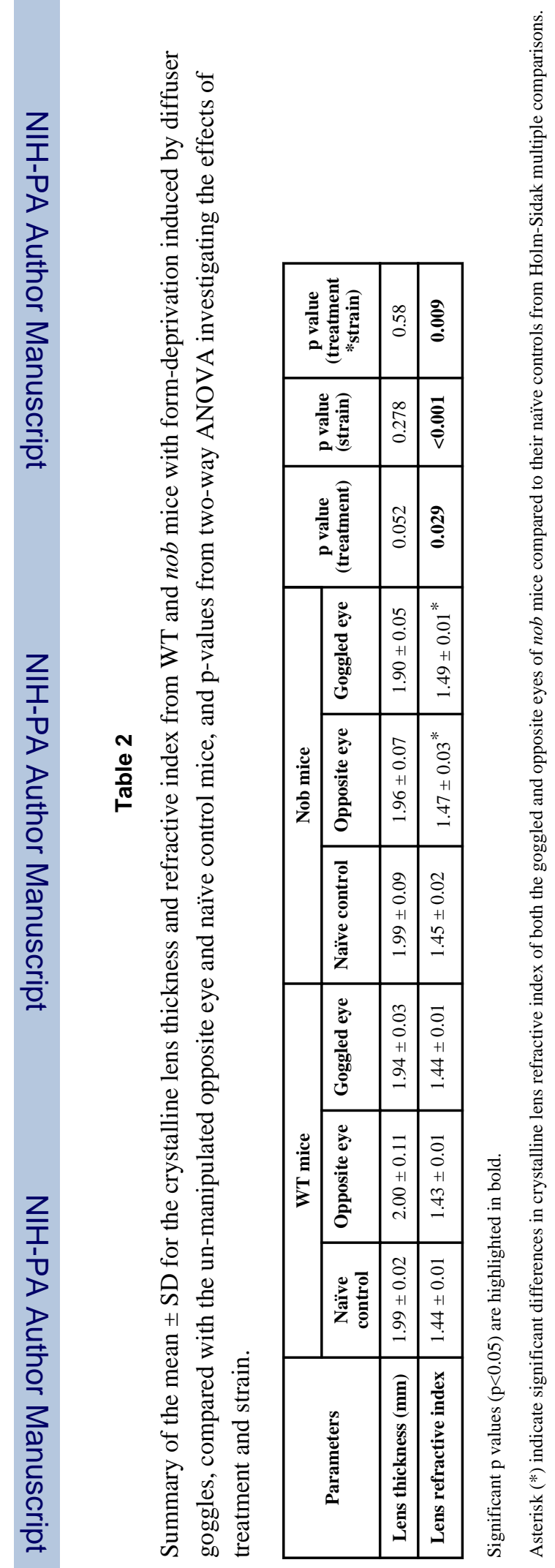

Exp Eye Res. Author manuscript; available in PMC 2015 August 01. 